Bolm Inst. oceanogr., S Paulo, 31(2):43-55, 1982

\title{
SPHAEROMATIDAE (ISOPODA: FLABELLIFERA) DA ZONA ENTRE-MARÉS E FUNDOS RASOS DOS ESTADOS DE SÃO PAULO E RIO DE JANEIRO
}

\author{
Ana Maria Setubal PIRES \\ Instituto Oceanogräfico da Universidade de São Paulo
}

Synops is

The present work deals with twelve species of Sphaeromatidae from several substrata in the intertidal and shallow infralittoral zone in São Paulo and Rio de Janeiro States, Brazil. Sand, stones, empty tests of barnacles, empty tubes of polychaete, byssus of mussels, oyster beds, macroscopic algae and sea grass (Spartina alternifloral were collected and analysed. The following nomenclaturai changes have been made: Pseudosphaeroma jakobii and Exosphaeroma platense are synonymyzed with Cassidinidea tuberculata; Pseudosphaeroma rhombofrontalis is placed in the genus Tholozodium; Paradynoides brasiliensis is synonymyzed with Dynoides castroi and Dynamenella antonii with D. tropica. A key to species, the geographical distribution and notes on the habitat of the species are also fumished.

Introdução

Os costões rochosos são povoados por numerosos animais, notadamente por crustáceos, entre os quais são comuns os Isopoda. Nas regiōes em que o substrato se apresenta coberto por algas, a fauna de crustāceos existente é rica e diversificada (Hagerman, 1966; Dommasnes, 1969; Mukai, 1971) e os isōpodes estão presentes, muitas vezes, em nümero bastante elevado.

Entretanto, nos costões rochosos, não só as algas são habitadas por estes crustáceos. Outros tipos de substratos, tais como as cavidades formadas por carapaças vazias de cirrípedes, os espaços entre o bisso de moluscos e tubos de poliquetos, são tambëm explorados, especialmente pelos Sphaeromatidae.

Os fundos inconsolidados da região entre-marés e infralitoral raso são tambēm povoados por vārias espēcies de Isopoda, estando os Sphaeromatidae bastante bem representados dentro da comunidade aí existente. Estes isópodes podem viver, pois, numa diversidade muito grande de habitats, tolerando uma larga faixa de variações das condições ambientais, tais como temperatura, salinidade, dessecação e batimento das ondas. Este fato é comprovado pelo número relativamente grande de gêneros e de espēcies que têm sido encontrados por alguns autores em diferentes regiões do mundo (Menzies \& Glynn, 1968; Jansen, 1971; Harrison \& Holdich, 1982).

Publ. n! 542 do Inst. oceanogr. da Usp.
0 presente estudo tem por objetivos fornecer: a classificação atual dos Sphaeromatidae encontrados; as novas ocorrências das espécies nos Estados de São Paulo e Rio de Janeiro, nas zonas entre-marés e submersa rasa; a distribuição das espēcies nos diferentes substratos; e os dados biológicos referentes às mesmas. E uma atualização e complementação do primeiro levantamento dos Sphaeromatidae brasileiros, realizado por Loyola e Silva (1960).

Material e mëtodos

Os Sphaeromatidae estudados são, em sua maioria, provenientes de coletas realizadas no 1itoral dos Estados de São Pau1o e Rio de Janeiro, durante o período compreendido entre 1972 e 1982. Incluídos neste trabalho estão também alguns exemplares que me foram encaminhados para determinação. Em São Paulo, o material foi obtido nas seguintes localidades: Ubatuba, São Sebastião, Bertioga, Guarujā, Santos, Itanhaém, Peruíbe e Cananéia. No Rio de Janeiro, em Būzios, Cabo Frio, Arraial do Cabo, Angra dos Reis e Parati.

Os seguintes tipos de substratos foram analisados no presente estudo: carapaças vazias de Cirripedia, bisso e conchas de mexilhões, colônias de Phragmatopoma, algas, areia, fendas nas rochas, face inferior de pedras e bancos de ostras.

As carapaças vazias de Tetraclita squamosa e Chthamalus sp. foram quebra- 
das individualmente, com auxilito de espátula. Os animais foram coletados com pinça, contados e colocados em vidros numerados. No costão do Lagosteiro, Ubatuba, as oito amostras obtidas foram retiradas de uma área delimitada com um quadrado de $20 \times 20 \mathrm{~cm}$. Os isópodes presentes em cada carapaça de cirrípede foram colocados em vidros numerados, para posterior contagem e determinação do sexo e grau de desenvolvimento.

Colônias do poliqueto Phragmatopoma lapidosa foram coletadas, colocadas em cubas plásticas para posterior fragmentação e retirada dos isōpodes.

Os mexilhões, Brachidontes darwinianus e $B$. solisianus, foram raspados da rocha com espátula e colocados em sacos plásticos rotulados, para posterior triagem dos isópodes existentes entre o bisso dos moluscos.

Cerca de $500 \mathrm{~g}$ de algas macroscópicas bênticas foram coletadas manualmente e colocadas em sacos plásticos, para posterior lavagem e retirada dos animais em laboratório. A lavagem se fez com àgua doce acrescida de algumas gotas de formol neutralizado.

Os isópodes foram fixados com formol $4 \%$ por cerca de 1 hora e, depois, transferidos para älcool $70 \%$.

Os animais examinados se encontram depositados na coleção de referência existente no Instituto Oceanogräfico da Universidade de São Paulo.

A distribuição geogräfica das espécies é, sempre, dada do norte para o Sul.

Resultados

As espécies de Sphaeromatidae encontradas nos vários substratos amostrados são em número de 12 , pertencentes a 11 gêneros. Damos a seguir uma chave para a identificação dessas espécies.

Chave para os Sphaeromatidae do litoral dos Estados de São Paulo e Rio de Janeiro:

1. Pleópodos 4 e 5 com exópodo e endópodo opaco, carnoso, com dobras branquiais (Fig. 23) ......Subfamília Dynameninae Bowman, 1981

- Pleópodos 4 e 5 com exópodo e endópodo transparente, ou com exópodo transparente e endópodo opaco ........... 2

2. P1eópodos 4 e 5 com endópodo opaco, carnoso, com dobras branquiais e exópodo transparente, sem dobras
(Fig. 5) $\ldots \ldots \ldots \ldots$. Subfamília Sphaeromatidae Milne Edwards, 1840

- Pleópodos 4 e 5 com endópodo e exópodo transparentes, com dobras fracas, não branquiais (Figs 9-10, 16-17) ...... Subfamília Cassidininae Iverson, 1982

3. Margem distal do pleotelson com projeção triangular mediana, superfície dorsal com 2 carenas longas, longitudinais, apresentando a parte posterior mais alta que a anterior (Fig. 1) .......... Cymodoce brasiliensis

- Margem distal do pleotelson inteira ou com reentrância ou fenda ..... 4

4. Pleotelson com reentrância mediana ................ Dynoides castroi

- Pleotelson com margem distal inteira $\ldots \ldots \ldots \ldots \ldots \ldots \ldots \ldots \ldots \ldots \ldots \ldots$

5. Margem distal do pleotelson arredondada (Fig. 6) ...... Sphaeroma walkeri

- Margem distal do pleotelson triangular (Fig. 4) ...... Sphaeroma terebrans

6. Corpo deprimido dorsoventralmente, margens externas ciliadas, basiendopodito do urópodo 2,5 vezes mais 1ongo que exópodo ......... 7

- Corpo não deprimido, enrolando-se como bola, urópodos subiguais no comprimento, pereópodos munidos de grande quantidade de cerdas longas ....... ........ Tholozodium rhombofrontalis

7. Cabeça imersa no pereonito I 1áteroposteriormente; epístoma grande, ciliado, visivel dorsalmente entre as antenas (Fig. 7) ...... Dies fluminens is

- Cabeça não imersa no pereonito I; epístoma pequeno, visível dorsalmente separando as antenas ..... Cassidinidea tuberculata

8. Pleotelson apresentando margem distal tubiforme com orifício central; superfície dorsal microtuberculada; seis tubérculos grandes, semi-esféricos, presentes no pleon (Fig. 18) ... ........... Cymodocella guarapariens is

- Margem distal do pleotelson lisa, ou com fenda, não tubiforme ....... 9

9. Machos e fêmeas com margem distal do pleotelson 1 isa, arredondada, sem fenda; urópodos estreitos (Figs 24-25). Corpo liso, sem tubérculos .......... 


\section{Sphaeromopsis mourei}

- Machos e fêmeas com acentuado dimorfismo sexual. Margem distal do pleotelson, no macho, com fenda grande e profunda, na fêmea, com fenda pequena e rasa; urópodos muito alargados ou muito longos nos machos, e mais estreitos e curtos nas fêmeas. ...................... 10

10. Corpo com pequenos tufos de cerdas dorsalmente. Machos apresentando margem distal do pleotelson com fenda larga em toda a sua extensão, parte basal com um dente central e dois submedianos; urópodos estreitos, com exópodo muito 1ongo e curvo para dentro, ultrapassando muito o äpice do pleotelson (Fig. 26). Pleotelson da fêmea com 3 grandes tubérculos transversais; margem distal com pequena reentrância semicircular, endópodo do urópodo distalmente truncado (Fig. 27) ...........Paracerceis sculpta

- Corpo sem tufos de cerdas. Urópodos lamelares, subiguais na largura, exópodo cerca de $3 / 4$ do comprimento do endópodo ................ 11

11. Dorso do pleotelson com tubérculos semi-esféricos, sendo em número de 4 na fêmea e 5 no macho; macho adulto com forâmen cordiforme, 1 igado ao ápice por fenda estreita e curta (Figs 21-22) ...... Paradella dianae

- Dorso do pleotelson com microtubérculos formando um " $V$ " invertido, mais marcante nos machos adultos; macho adulto com forâmen cordiforme ligado ao ápice por fenda estreita e longa (Figs 19-20)..... Dynamenella tropica

\section{Subfamilia Sphaeromatinae}

Cymodoce brasiliensis Richardson, 1906 (Fig. 1)

Esta espécie ocorreu em Phragmatopoma lapidosa, em pequeno número, e em algas, desde a zona entre-marés até 5-6 m de profundidade. A maior densidade verificada foi de 43 indivíduos por $500 \mathrm{~g}$ de Sargassum cymosum. Cymodoce brasiliensis é comumente encontrada em águas costeiras rasas. Richardson (1906) relata que a espécie foi coletada a $36 \mathrm{~m}$ de profundidade, em conchas quebradas, e Loyola e Silva (1960) obteve-a em algas e em Porifera, desde a zona entre-marés até $5 \mathrm{~m}$ de profundidade.

\section{Distribuição geográfica}

BRASIL. Rio Grande do Norte: Cabo de são Roque (localidade-tipo); Pernambuco: Recife; Bahia: Salvador; São Paulo: Ubatuba, Santos, Itanhaém, Peruỉbe; Paranā: Caiobá, Matinhos, Ilha do Mel; Santa Catarina: Ponta das Canas, Camboriū, Piçarras, Itajaî, Florianōpolis. As ocorrências novas aqui registradas são as do Estado de São Paulo e as de Itajaí e Florianópolis, noEstado de Santa Catarina.

\section{Material examinado}

São Paulo: Itanhaém (1976, A. M. S. Pires col., 18 exemplares); Santos (Ilha Porchat, em Ulva sp., 20.10.1979, S. A. Vanin col., 3 exs.); São Sebastião (Praia do Cabelo Gordo, 09.09.1972, A. M. S. Pires col., 13 exs.); Ubatuba (Enseada do Flamengo, em algas do médio litoral inferior, abril e setembro de 1975, A. M. S. Pires e S. A. Vanin co1., 77 exs.); idem (Lagosteiro, 1972, A. M. S. Pires col., 10 exs.); idem (Praia do Flamenguinho, em algas, 5-6 m de profundidade), 1965 , P. S. Moreira co1., 33 exs.). Santa Catarina: Itajaí (Praia Vermelha, em algas Rodofíceas, 12.04.1979, S. Ditadi co1., 2 exs.); Florianópolis (Praia da Armação, 17.05.1978, F. Santos Filho col., 1 ex.).

Dynoides castroi Loyola e Silva, 1960 (Figs 2-3)

Dynoides castroi Loyola e Silva, 1960:91. Paradynoides brasiliensis Loyola e Silva, 1960: 101. Sinônimo novo.

Dynoides castroi foi encontrado, atē o presente, somente na região entre-marés. Ocorreu nos tubos vazios de Phragmatopoma lapidosa, junto a Sphaeroma walkeri e Dynamenella tropica. Na Enseada do Flamengo, Ubatuba, foi obtido em bancos de Phragmatopoma, junto a Cirolana parva; em carapaças vazias de Tetraclita squamosa e Chthamalus sp., dominando sozinho este ambiente; e em vārias espécies de algas.

Um estudo mais detalhado sobre $D$. castroi no ambiente das carapaças vazias de Tetraclita squamosa foi realizado em 16.01.1976, no costão do Lagosteiro, Enseada do Flamengo, Ubatuba. A maioria das carapaças apresentou-se coberta pela alga incrustante, Ralfsia expansa. Das oito amostras coletadas ( 3 da secção 
Bolm Inst. oceanogr., S Paulo, 31(2), 1982

vertical e 5 da horizontal), retirou-se 49 carapaças, onde foram encontrados 94 Dynoides castroi. Foram obtidos de 1 a 4 animais por carapaça; em $65 \%$ das mesmas, foi constatado haver um adulto com 1 a 3 jovens; em $35 \%$ das carapaças, encontrou-se apenas jovens.

No costão, com o abaixamento da maré, foi observada a migração de alguns exemplares de $D$. castroi para carapaças situadas em niveis inferiores. A movimentação dos animais dentro da faixa de Tetraclita ou entre esta e o cinturão de algas situado logo abaixo, se deu sempre no momento do refluxo de uma onda, quando da formação de uma esteira de água sobre a rocha.

Um outro aspecto estudado em relação à populaçaõ de 0 . castroi foi o da densidade de individuos por tipo de substrato coletado. Em setembro de 1975, foram obtidas amostras de algas e de carapaças de Tetraclita squamosa em três costoes da Enseada do Flamengo, Ubatuba: Ponta Grossa, Ponta do Espia e

Lagosteiro. Verificou-se que aproximadamente $75 \%$ da população de $D$. castroi amostrada em algas é formada por jovens, e o restante, $25 \%$, formado por fêmeas e machos imaturos. Ao conträrio, nas carapaças de Tetraclita squamosa, $73 \%$ da população amostrada é composta de adu1tos em reprodução, estando os machos em maior proporção (razão sexual de $1: 1,5$ ). Estes dados sugerem uma localização preferencial dos jovens em algas e, dos adultos, nas carapaças vazias de Tetraclita squamosa (Tab. 1).

\section{Distribuição geogräfica}

BRASIL. Rio de Janeiro: Cabo Frio, Arraial do Cabo, Barra de Guaratiba. São Paulo: Ubatuba, Guarujā, Santos, Itanhaém. São todas ocorrências novas, com exceção daquela de Barra de Guaratiba. A presente distribuição amplia para o sul

Tabela I - Número e proporção de jovens e adultos de Dynoides castroi em algas e carapaças vazias de Tetraclita squamosa (costão do Lagosteiro, Ubatuba, janeiro de 1976)

\begin{tabular}{|c|c|c|c|c|c|c|}
\hline Substrato & $\begin{array}{l}N ? \\
\text { jovens. }\end{array}$ & $\begin{array}{l}N ! \\
\text { adul tos }\end{array}$ & Total & jovens. & adul tos & Total \\
\hline Algas & 863 & 292 & 1155 & 74.7 & 25.3 & 100,0 \\
\hline $\begin{array}{l}\text { Tetraclita } \\
\text { squamosa }\end{array}$ & 74 & 200 & 274 & 27,0 & 73,0 & 100,0 \\
\hline
\end{tabular}

- limite de distribuição da espécie. Dynoides castroi foi encontrado em värias algas (Amphiroa beauvoisii, Cheilosporum sp., Sargassum cymosum, Enteromorpha clathrata, Ulva lactuca) em carapaças vazias de Chthamalus sp. e Tetraclita squamosa, em bancos de mexilhão e em tubos vazios de Phragmatopoma lapidosa.

\section{Material examinado}

Rio de Janeiro: Cabo Frio (boca do Canal de Itajuru, 12.02.1980, A. W. S. Pires e S. A. Vanin col., 8 exs.); idem (Praia do Forte, 11.02.1980, A. M. S. Pires e S. A. Vanin col., 100 exs.); Arraial do Cabo (Prainha, em Chthamalus sp., local abrigado, 13.02.1980, A. M. S. Pires e S. A. Vanin col., 2 exs.); idem (Praia do Boqueirão, em banco de mexi1hão, 14.04.1977, F. Fernandes col., 33 exs.); idem (Praia do Forno, em banco de mexilhão, 12.04.1977, F. Fernandes col., 3 exs.). São Paulo: Ubatuba (Enseada do Flamengo, em algas, 1965, P. S. Moreira col., 6 exs.); idem (Enseada do Flamengo, abril e setembro 1975, A. M. S. Pires e S. A. Vanin col., 1972 exs.); Santos (Ilha Porchat, em Phragmatopoma lapidosa, 14.11.1978, A. M. S. Pires e S. A. Vanin col., 78 exs.); idem (I1ha das Palmas, 09.09.1980, A. M. S. Pires col., 9 exs.); idem (Ilha de Lajes, em algas, setembro 1976, P. S. Moreira col., 86 exs.); Itanhaëm (17.03.1976, A. S. Tararam col., 42 exs.); idem (Praia do Poço, 13.05.1980, A. S. Tararam co1., 28 exs.); Guarujā (Saco do Major, 1974, A. M. S. Pires col., 8 exs.).

\section{Discussão}

o gênero Paradynoides Loyola e Silva, 1960 foi recentemente sinonimizado com Dynoides Barnard, 1914, por Bruce (1980). Dynoides castroi foi descrito com base em um único exemplar macho e, na época, não se pensou ser esta uma espécie sexualmente dimórfica. Assim sendo, Paradynoides continha, na realidade, exemplares jovens, fêmeas e machos imaturos de Dynoides castroi. Bruce (1980) suspeitou da identidade de Paradynoides ao comparar as fêmeas e machos imaturos de Dynoides dentisinus Shen, 1929 e $D$. brevispina Bruce, $1980 \mathrm{com}$ as figuras de Paradynoides (Loyola e Silva, 1960, figs 16-17).

Porēm, Bruce (op.cit.) fez somente a sinonímia do gênero Dynoides, não se re- 
ferindo ao "status" de Paradynoides

brasiliensis: se esta seria uma espécie vālida ou se seria sinônimo de outra já descrita. Assim sendo, tendo sido encontrados em Cabo Frio (RJ), machos de Dynoides castroi e fêmeas de Paradynoides brasiliensis cohabitando a mesma carapaça vazia de Chthamalus sp., e havendo sido realizado o estudo morfológico das duas espécies, pode-se concluir que $P$. brasiliensis è sinônimo de $D$. castroi.

Um fato curioso que acontece em Dynoides castroi é quanto à segmentação do exópodo do plépodo 3. Nos jovens, fêmeas e machos imaturos, esse ramo é biarticulado, enquanto que, nos machos adultos, è uniarticulado. Este fato, provavelmente também contribuiu para que Loyola e Silva (1960) criasse um gênero e uma espécie nova (Paradynoides brasiliensis) para colocar os jovens, fêmeas e machos imaturos de Dynoides castroi.

\section{Sphaeroma terebrans Bate, 1866 (Fig. 4)}

Sphaeroma terebrans foi coletado em madeira encontrada na região entre-marés e banhada por água salobra. A espécies é bastante comum nesse ambiente, pois perfura madeira e nela constrói galerias.

Distribuição geogrāfica

BRASIl. São Paulo: Cananéia; Santa

Catarina: Joinville, Piçarras. MOÇAMBIQUE. ĀFRICA DO SUL.

Material examinado

São Paulo (Cananēia, 10.01.1981, J. Abreu col., 9 exs.).

\section{Sphaeroma walkeri Stebbing, 1905}

$$
\text { (Figs 5-6) }
$$

Sphaeroma walkeri foi a espēcie dominante em bancos de Phragmatopoma lapidosa. Num bloco de tubos vazios de cerca de 1 quilo, foram obtidos 193 exemplares de S. walkeri, seguindo-se em abundância, Dynoides castroi (78 exemplares), Paradella dianae (35 exs.), Dynamenella tropica (3 exs.) e Sphaeromopsis mourei (1 ex.). Sphaeroma walkeri foi tambēm obtido em carapaças vazias de Tetraclita squamosa. Não foi encontrado em algas $e$, embora tenha sido assinalada a presença da espécie em Ubatuba (Loyola e Silva, 1960), não se coletou nenhum exemplar da mesma nas várias praias in- vestigadas.

Distribuição geográfica

CEILÃO. INDIA. EGITO. ĀFRICA DO SUL. AUSTRÁLIA. PORTO RICO. BRASIL. Ceará: Fortaleza; Pernambuco; Rio de Janeiro; São Paulo: Ubatuba, Guarujā, Santos, Peruibe; Parană: Ilha do Mel. As ocorrências novas são as de Peruîbe, Santos e Guarujā.

\section{Material examinado}

São Paulo: Santos (Ilha de Urubuqueçaba, em banco de ostra, junto com Paradella dianae, 05.1977, 1 ex.); Santos (Ilha Porchat, em Phragmatopoma lapidosa, 07.10.1976, S. A. Vanin col., 14 exs.; idem, 14.11.1978, A. M. S. Pires e S. A. Vanin co1., 193 exs.; idem, 20.10.1979, S. A. Vanin col., 39 exs.); Santos (Ilha das Palmas, em Tetraclita squamosa, 09.09.1980, A. M. S. Pires col., 3 exs.); Guarujā (Costão do Saco do Major, 1977, A. M. S. Pires col., 20 exs.); idem (Praia das Pitangueiras, em Phragmatopoma lapidosa, 24.01.1980, A. M. S. Pires e S. A. Vanin col., 119 exs.); Peruỉbe (em Phragmatopoma lapidosa, 01.1976, M. Scaico col., 20 exs.).

\section{Subfamilia Cassidininae}

Dies fluminensis (Mañe-Garzon, 1944)

$$
\text { (Fig. 7) }
$$

Dies fluminensis ocorreu em Spartina alterniflora, na zona do mediolitoral, em salinidade entre 30 e $32 \%$. E uma espécie eurihalina, no Paraná foi coletada por Loyola e Silva (1960) em salinidade de $17 \%$, vivendo em águas tropicais e subtropicais da costa sudeste brasileira.

\section{Distribuição geogrāfica}

BRASIL. Rio de Janeiro: Lagoa de Camorim (localidade-tipo), Lagoa da Tijuca; São Paulo: Cananéia; Paranā: Baĩa de Guaratuba; Santa Catarina: Camboriū; Rio Grande do Sul: Lagoa dos Patos, Canal de São Gonçalo. A espécie é assinalada pela primeira vez para o litoral paulista.

\section{Material examinado}

São Paulo: Cananéia (15.09.1981, A. S. Tararam, A. M. Takeda e Y. Wakabara col., 5 exs.). 

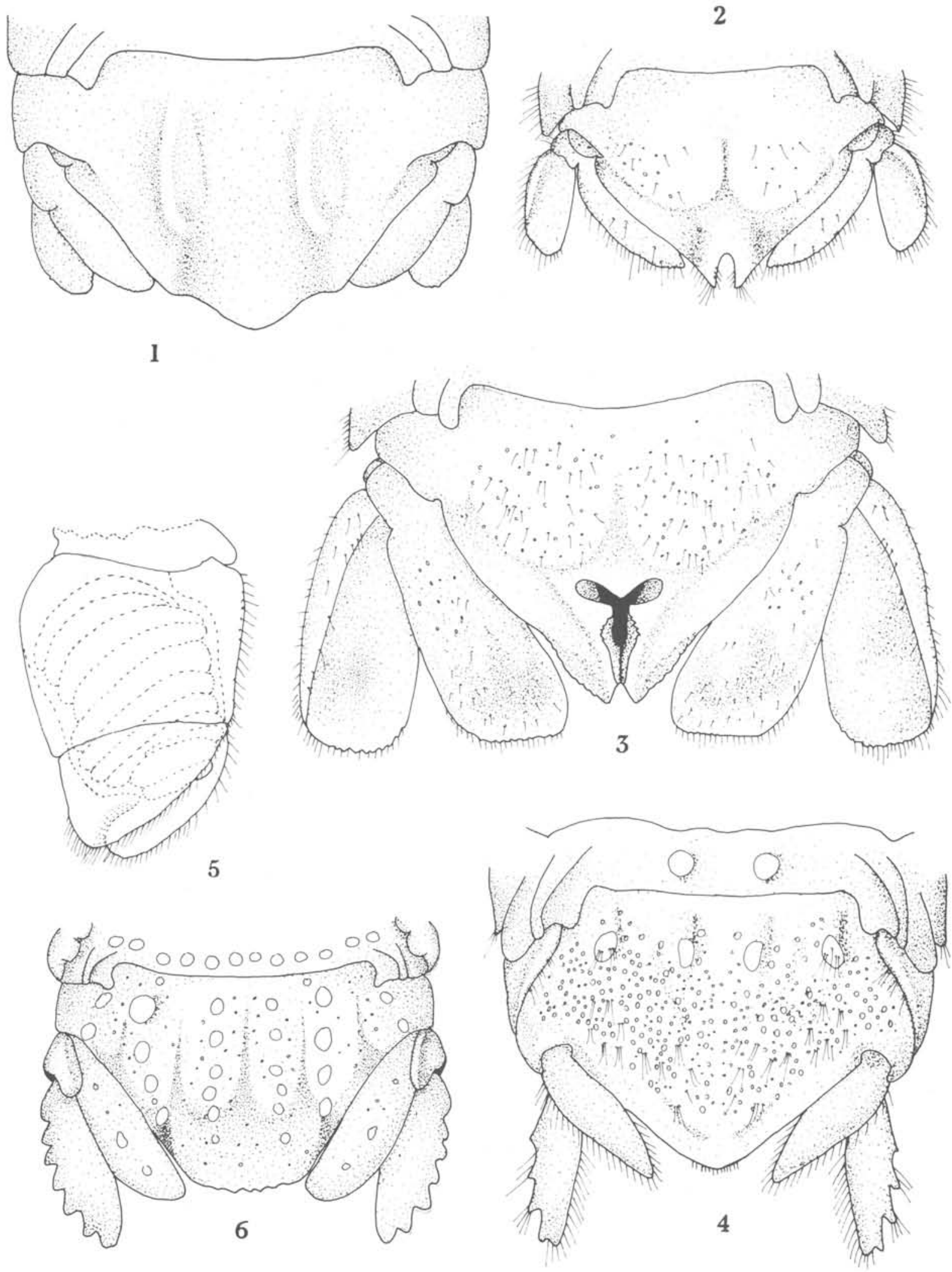

1. Cymodoce brasiliensis, fêmea, $5,0 \mathrm{~mm}$ : pleotelson. 2-3. Dynoides castroi; 2. fêmea, 3,8 mm: pleotelson; 3. macho, 5,1 mm: pleotelson. 4. Sphaeroma terebrans, macho, 8,3 mm: pleotelson. 5-6. Sphaeroma walkeri, fêmea, 8,0 mm; 5. pleópodo 4; 6 . pleotelson. 
Cassidinidea tuberculata Richardson, 1912 (Figs 8-10)

Cassidinidea tuberculata Richardson, 1912: 107-108.

Exosphaeroma platense Giambiagi, 1922: 234. Sinônimo novo.

Pseudosphaeroma jakobii Loyola e Silva, 1959:79. Sinônimo novo.

Esta espécie foi coletada em raízes de Spartina alterniflora e em areia, desde a região entre-marés até $1-1,5 \mathrm{~m}$ de profundidade. Segundo Loyola e Silva (1960), ela pode ser encontrada sob pedras e em vegetação aquática, em ägua marinha ou mais frequentemente salobre.

\section{Distribuição geogrāfica}

MEXIC0. Madre, Maron Lagoon. BRASIL. Rio de Janeiro; São Paulo: Cananéia; Paranâa: Baía de Guaratuba, Matinhos; Santa Catarina: Joinville; Rio Grande do Sul: Rio Grande (porto), Canal de São Gonçalo, Pelotas (porto). ARGENTINA. Buenos Aires: rio Santiago.

Tholozodium rhombofrontalis (Giambiagi, 1922) comb. nov.

$$
\text { (Figs 11-17) }
$$

Exosphaeroma rhombofrontalis (Giambiagi, 1922: 237.

Pseudosphaeromarhombofrontalis; Loyola e Silva, 1960: 115.

Espécie muito abundante na zona entre-marés, especialmente na areia entre as raízes de Spartina alterniflora. Foi obtida também, mas em menor quantidade, no infralitoral raso $(1-1,5 \mathrm{~m})$, em fundo de areia. São animais característicos de litoral arenoso.

\section{Distribuição geogräfica}

BRASIL. São Paulo: Cananēia; Paranā:
Ilha do Me1. ARGENTINA. Buenos Aires: Quequén. Com o presente trabalho o 1imite de distribuição da espécie fica estendido mais para o norte: Cananéia, São Paulo.

\section{Material examinado}

São Paulo: Cananéia (em Spartina alterniflora, entre-marés, 25.08.1981, A. M. Takeda, A. S. Tararam eY. Wakabara co1., 793 exs.); idem (idem, 15.09.1981, A. M. Takeda, A. S. Tararam eY. Wakabara col., 438 exs.); idem (idem, 20.10.1981, A. M. Takeda, A. S. Tararam Y. Wakabara col., 706 exs.); idem (em areia, entremarés, 10.02.1982, A. S. Tararam col., 20 exs.); idem (em areia, $1 \mathrm{~m}$ de prof., 26.08.1981, A. M. Takeda, A. S. Tararam e Y. Wakabara col., 3 exs.).

\section{Discussão}

Tholozodium rhombofrontalis era conhecido no Brasil através de um único exemplar macho proveniente do Paraná. Até o presente nada se sabia sobre o dimorfismo sexual desta espécie, pois na descrição original nada consta a respeito. Com o material muito numeroso à disposição, nos foi possível comparar ambos os sexos e encontrar dimorfismo sexual, pouco acentuado, nos pereópodos. 0 pereópodo I, tanto em machos quanto em fêmeas, difere dos demais por apresentar um número bem menor de cerdas em todos os artículos. Os pereópodos II-VII nos machos, apresentam as margens distais do ísquio, mero e carpo com um número bem maior de cerdas em relação às fêmeas; os artîculos mero, carpo e própodo possuem, na face ventral, uma ärea densamente pilosa nos machos, que é inexistente nas fêmeas (Figs 13-15). Os ovos se desenvolvem numa bolsa interna no corpo da fêmea.

A espécie que ocorre em nosso litoral apresenta quase todas as características do gênero Tholozodium Eleftheriou, Holdich \& Harrison, 1981. Em T. rhombofrontalis não hā vestígio de sutura das placas coxais nos pereonitos, a bolsa marsupial interna é única, e a margem distal do pleotelson apresenta uma reentrância bastante rasa, melhor observada em vista 1átero-dorsal. Apesar dessas diferenças, preferimos deixar rhombofrontalis em Tholozodium, jā que é o gênero do qual mais se aproxima. 

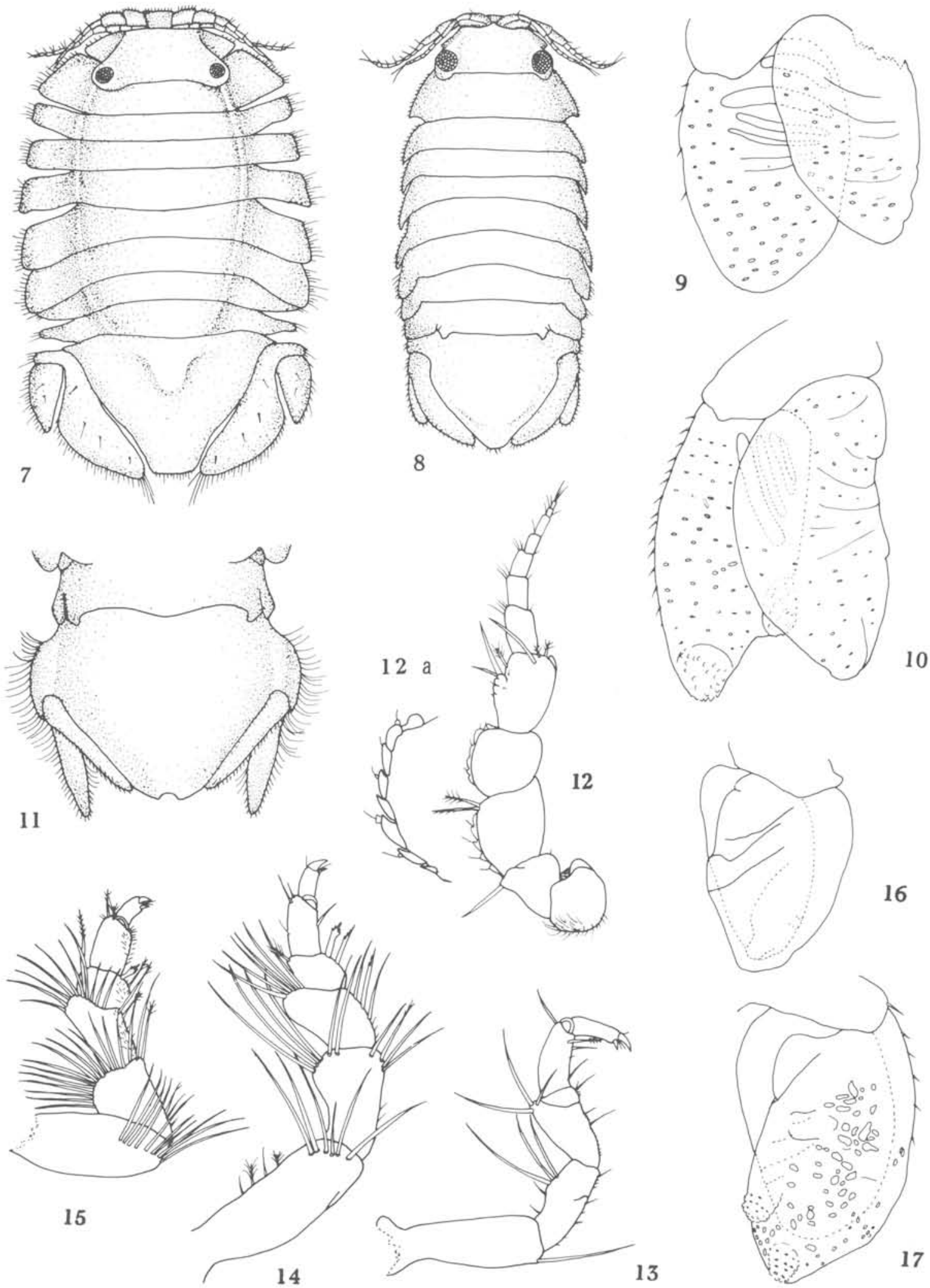

7. Dies fluminensis, macho, 2,8 mm: vista dorsal. 8-10. Cassidinidea tuberculata, fêmea, 5,1 mm: pleotelson; 8. vista dorsal; 9. pleópodo 4; 10. pleöpodo. 11-17. Tholozodium rhombofrontalis; 11-14. fêmea, 3,9 mm: 11. pleotelson; 12. antena 2; 12 a. escamas da margem externa da antena $2 ; 13$. pereópodo 1; 14 . pereópodo 111 ; 15-17. macho, 3,5 mm: 15. pereöpodo 1; 16. pleōpodo 4; 17. pleópodo 5 . 
Subfamilia Dynameninae

Cymodocella guarapariensis Loyola e Silva, 1965

$$
\text { (Fig. 18) }
$$

Esta espécie foi encontrada em Arraial do Cabo (RJ), em banco de mexilhão, nos espaços entre o bisso dos animais e a areia que se deposita sobre o substrato. Até o presente, Cymodocella

guarapariensis só era conhecida da localidade-tipo, Guarapari (ES), tendo sido coletada em algas macrofíticas bênticas da região entre-marés (Loyola e Silva, 1965).

\section{Distribuição geogrāfica}

BRASIL. Espirito Santo: Guarapari; Rio de Janeiro: Arraial do Cabo. A nova ocorrência estende para o sul o limite de distribuição da espécie.

\section{Material examinado}

Rio de Janeiro: Arraial do Cabo (Boqueirão, 10.01.1977, F. Fernandes co1., 2 exs.).

Dynamenella tropica Loyola e Silva, 1960 (Figs 19-20)

Dynamenella tropica Loyola e Silva, 1960: 150 .

Dynamenella antonii Loyola e Silva, 1960; 160; Pires, 1981b: 29; Harrison \& Holdich, 1982: 90. Sinônimo novo.

Esta espécie habita diversos substratos: tubos vazios de Phragmatopoma lapidosa, bancos de Crassostrea rhizophorae, areia entre o bisso dos mexilhões Brachidontes darwinianus e $B$. solisianus, o sedimento acumulado na base das algas Enteromorpha flexuosa e Bryocladia cuspidata, carapaças vazias de Chthamalus sp.. São animais que preferem praias abrigadas. Em Cabo Frio, obteve-se Dynamenella tropica nas carapaças localizadas na zona mais a1 to do mediolitora1, enquanto Paradella dianae foi obtida no mesmo costão, porém em carapaças vazias situadas numa zona mais baixa do mediolitoral. Ambas as espécies apresentaram densidade relativamente igual: 200 exemplares de $D$. tropica e 205 de P. dianae.

Observou-se que as carapaças vazias de Chthamalus sp., por serem pequenas, abrigavam somente um adulto, geralmente macho. Já nas carapaças de Tetraclita squamosa, maiores, encontrou-se machos, fêmeas e jovens, sendo mais freqüentes fêmeas com jovens. 0 número ce adultos foi maior que o de jovens: 169 contra 31 , o que dá uma freqüência de $84,5 \%$ de adultos vivendo em carapaças vazias de cirrípedes. D. tropica havia sido anteriormente encontrada na região entremarés, em rachaduras de trapiche de madeira e entre ostras e mexilhões

(Loyola e Silva, 1960).

\section{Distribuição geogrāfica}

BRASIL. Cearā: Fortaleza; Rio de Janeiro: Búzios, Cabo Frio, Mangaratiba; São

Paulo: Ubatuba, Santos, Peruíbe; Santa

Catarina: Porto Belo, Camboriū e

Picarras.

\section{Material examinado}

Rio de Janeiro: Búzios (Pr. Azeda, em Chthamalus, 12.02.1980, A. M. S. Pires e S. A. Vanin col., 200 exs.); Cabo Frio (entrada do canal de Itajuru, em Chthamalus sp., 10.02.1980, A. M. S. Pires e S. A. Vanin col., 1 ex.); São Paulo: Ubatuba (Enseada do Flamengo, $\mathrm{Pr}$. do Perequê-Mirim, abril e setembro 1975, A. M. S. Pires e S. A. Vanin col., 13 exs.); idem (Ubatumirim, Pr. do Estaleiro, em banco de ostra, abril 1977, G. Johnscher col., 3 exs.); Bertioga (Pr. do Indaiá, em banco de ostra, abril 1977, G. Johnscher col., 49 exs.); Santos (Ilha Porchat, em Phragmatopoma lapidosa, 14.11.1978, A. M. S. Pires e S. A. Vanin col., 3 exs.).

\section{Paradella dianae (Menzies, 1962)}

$$
\text { (Figs 21-23) }
$$

Dynamenopsis dianae Menzies, 1962: 341; Schultz, 1969: 123.

Dynamenella dianae: Menzies \& Glynn, 1968: 63, 113; Iverson, 1974: 166; Pires, $1980 \mathrm{~b}: 133$.

Paradella dianae: Harrison \& Holdich, 1982: 103.

Esta espécie foi encontrada em algas, banco de Phragmatopoma lapidosa, em banco de ostra e em carapaças vazias de Tetraclita squamosa e Chthamalus, sendo mais abundantes nestas ültimas. Quando sozinho dentro de seu abrigo, o macho adulto abre o pleotelson e bloqueia com ele a entrada da carapaça. Nessa posição, movimenta os pleópodos para promo- 
Bolm Inst. oceanogr., S Paulo, 31(2), 1982

ver a corrente de água necessāria para a respiração. Verificou-se que, nas carapaças menores (Chthamalus), geralmente havia um macho adulto, enquanto que as carapaças maiores (Tetraclita) eram habitadas por um macho com várias fêmeas, ou um macho com uma fêmea e värios jovens.

\section{Distribuição geogrāfica}

ESTADOS UNIDOS. Califörnia, Flórida; MEXICO. Baja Califórnia. PORTO RICO. BRASIL. Rio de Janeiro: Büzios, Cabo Frio e Arraial do Cabo; Sãa Paulo: São Sebastião, Bertioga, I1ha Porchat. AUSTRÁLIA. A ocorrência da espécie no 1itoral do Estado de São Paulo foi recentemente assinalada (Pires, 1980b) e, no presente trabalho, estendeu-se a distribuição também para o Estado do Rio de Janeiro. Paradella dianae ocorre em águas tropicais e ê bastante provável que venha a ser encontrada na costa nordeste brasileira, nas proximidades de portos.

\section{Material examinado}

Rio de Janeiro: Büzios (Pr. Azeda, em Chthamalus sp. e Tetraclita squamosa, 12.02.1980, A. M. S. Pires e S. A. Vanin col., 205 exs.); Cabo Frio (entrada do Canal de Itajuru, em Chthamalus sp., 10.02.1980, A. M. S. Pires e S. A. Vanin col., 23 exs.); Arraial do Cabo (em Chthamalus sp., 13.02 .1980 , A. M. S. Pires e S. A. Vanin col,, 20 exs.); São Paulo: São Sebastião (Pr. Flecheiras, em Chthamalus sp. e Tetraclita squamosa, dezembro 1978, 220 exs,); idem, 25.09 .1980 , A. M. S. Pires e S. A. Vanin col., 45 exs.); idem ( $\mathrm{Pr}$. do Segredo, A. M. S. Pires e S. A. Vanin col., 36 exs.); Santos (I1ha de Urubuqueçaba, em banco de ostra, maio 1977, G. Johnscher col., 35 exs.); idem (Ilha Porchat, 14.11.1978, A. M. S. Pires e S. A. Vanin col., 91 exs.); idem (idem 20.10.1979, A. M. S. Pires e S. A. Vanin col., 3 exs.); idem (Ilha das Palmas, em Chthamalus sp. e Tetraclita squamosa, 09.09.1980, A. M. S. Pires e S. A. Vanin col., 152 exs.).

Sphaeromopsis mourei (Loyola e Silva, 1960)

$$
\text { (Figs 24-25) }
$$

Pseudosphaeroma mourei Loyola e Silva,
1960: 138; Menzies \& Glynn, 1968: 66; Holdich \& Jones, 1973: 393; Pires, $19816: 29$.

Sphaeromopsis mourei, Holdich \& Harrison, 1981: 295.

Sphaeromopsis mourei foi encontrado na região entre-marés: em areia e bisso do mexilhão Brachidontes spp., na praia do Perequê-Mirim, Ubatuba, numa salinidade de 21,7 a $20,2 \%$; entre raízes de Spartina alterniflora e em macroalgas bênticas associadas com sedimento arenoso, em salinidade de $34 \%$. A espécie também ocorre em fundos rasos (6 m de prof.), em algas. Sphaeromops is mourei é uma espécie eurihalina, sendo encontrada em águas marinhas próximas à desembocadura de riachos de praias, sempre em grande quantidade.

\section{Distribuição geogräfica}

BRASIL. Pernambuco: Recife; Rio de Janeiro: Parati; São Paulo: Ubatuba, Santos, Cananéia; Santa Catarina.

\section{Material examinado}

Rio de Janeiro: Parati (Pr. do Guarda-Mor, coleta noturna de zooplâncton, 22.02.1982, R. Tsukamoto col., 6 exs.); Ubatuba (Enseada do Flamengo, em Sargassum cymosum, entre-marés, 1972, A. M. S. Pires col., 17 exs.); idem (idem, em algas e Brachidontes spp., entre-marēs, abril 1975, A. M. S. Pires e S. A. Vanin co1., 168 exs.); idem (Enseada da Fortaleza, Pr. do Lázaro, em Jania capillacea, 5-6 m de prof., outubro 1978, M. Flynn col., 5 exs.); Santos (Ilha Porchat, em Ulva sp. com Enteromorpha sp., 14.11.1978, S. A. Vanin col., 10 exs.); Cananéia (em Spartina alterniflora, 25.08 e 15.09.1981, A. M. Takeda, A. S. Tararam e Y. Wakabara col., 180 exs.); idem (em dragagem, 1-1,5 m de prof., 14.09.1981, A. M. Takeda e Y. Wakabara col., 6 exs.).

\section{Observações}

Sphaeromops is mourei apresenta um dimorfismo sexual pouco acentuado. Os machos possuem pleotelson relativamente mais estreito que as fêmeas: a proporção comprimento/largura é de aproximadamente $1,0: 1,4$ nos machos e $1,0: 2,0$ nas fêmeas (Figs 24 e 25). As fêmeas são, geralmente, maiores que os machos. Nestes, 


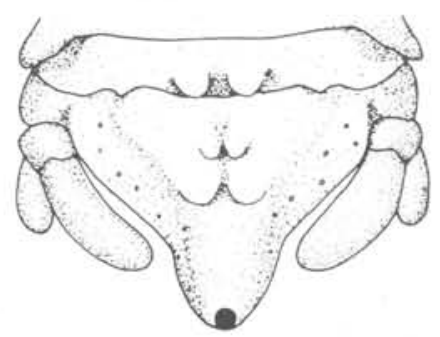

18
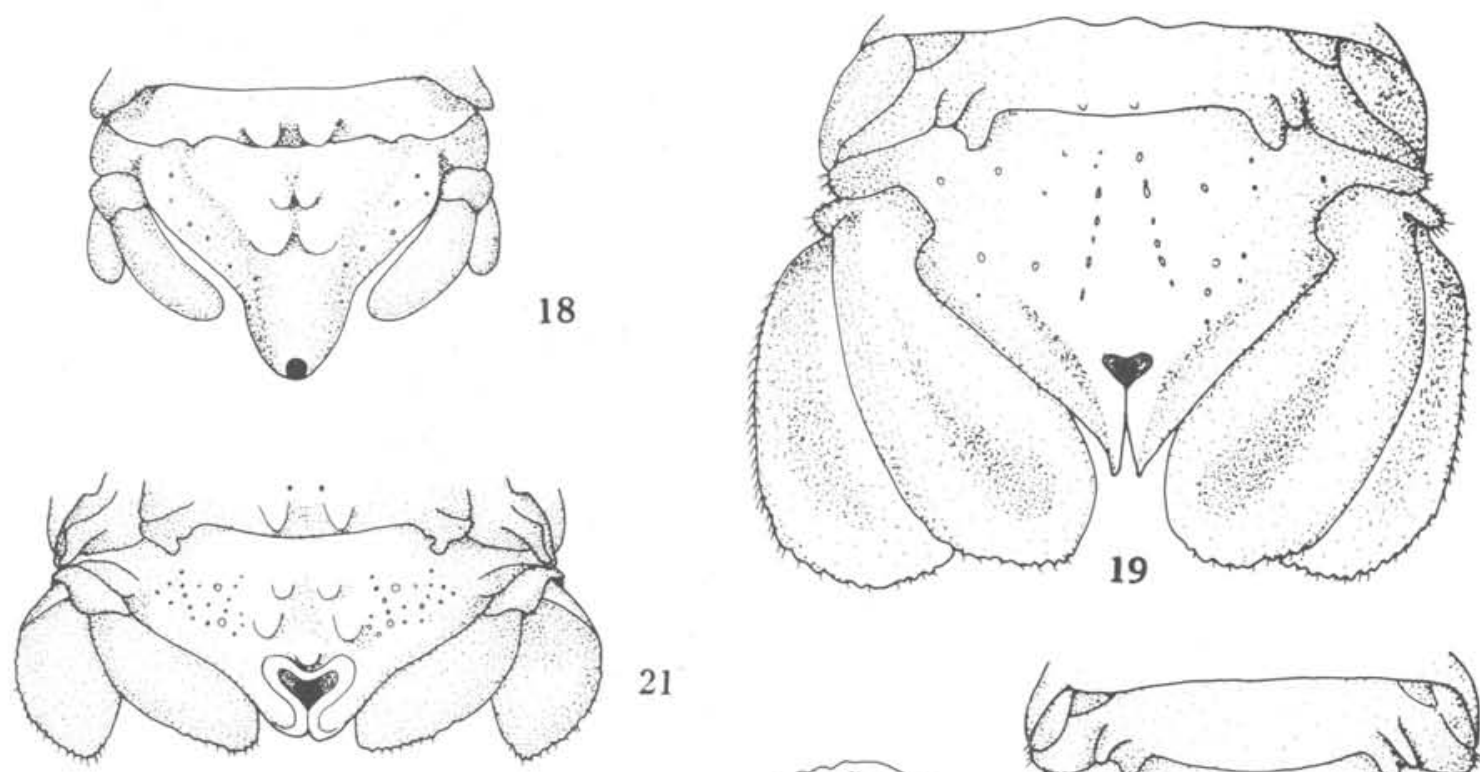

21

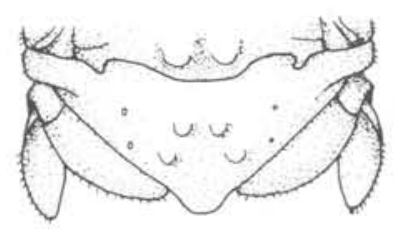

22

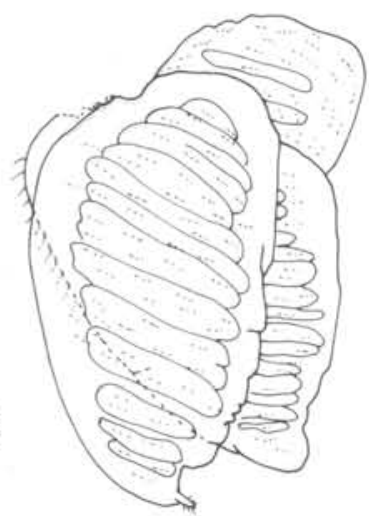

23

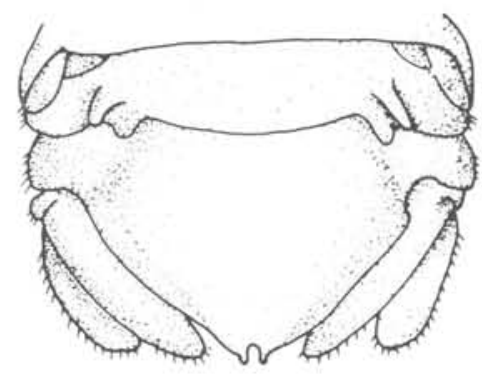

20
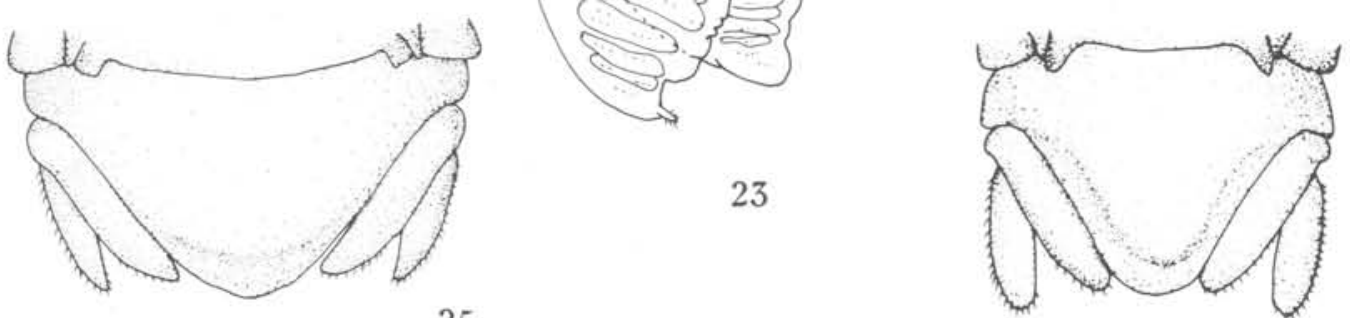

24
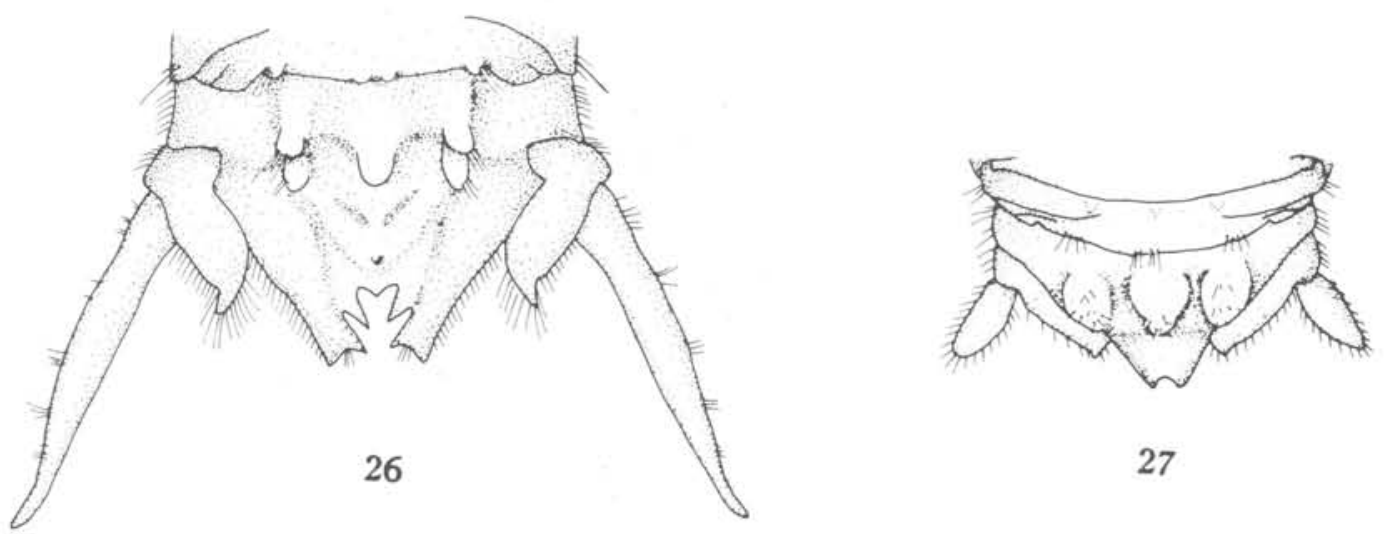

27

18. Cymodocella guarapariensis, fêmea com ovos, $2,8 \mathrm{~mm}$ : pleotelson. 19-20. Dynamenella tropica; 19. macho, 4,0 mm: pleotelson; 20. fêmea, 3,3: pleotelson. 2123. Paradella dianae; 21,23 . macho, $5,6 \mathrm{~mm}: 21$. pleotelson; 23 . pleópodo 4; 22 . fêmea, 4,1 mm: pleotelson. 24-25. Sphaeromops is mourei; 24 . macho, 2,7 mm: pleotelson; 25. fêmea, 3,3 mm: pleotelson. 26-27. Paracerceis sculpta; 26. macho, $6,4 \mathrm{~mm}$ : pleotelson; 27 . fêmea, 5,3 mm: pleotelson. 
os pereópodos II-VII apresentam-se revestidos por cerdas finas e longas; 0 exópodo do urópodo ultrapassa ligeiramente a margem distal do pleotelson, ao passo que nas fêmeas é mais curto.

Paracerceis sculpta (Ho1mes, 1904) (Figs 26-27)

Dynamene sculpta Holmes, 1904: 300. Cilicaea sculpta, Richardson, 1905: 318. Sergiella angra Pires, 1980a: 213; Pires, 1981a: 219.

A ocorrência desta espécie foi recentemente assinalada para o Brasil (Pires $1980 a ; 1981 a)$. Encontrou-se P. sculpta entre as algas Sargassum cymosum e Galaxaura sp., desde a zona entre-marēs até $5 \mathrm{~m}$ de profundidade, dentro de esqueleto de coral, sob pedras, e em carapaças vazias de Chthamalus sp. e Tetraclita squamosa. Richardson (1905) obteve P. sculpta em esponjas de água rasas e Resig (1978), em carapaças vazias de Balanus sp. e em algas.

\section{Distribuição geogrāfica}

ESTADOS UNIDOS. Califörnia: BRASIL. Rio de Janeiro: Angra dos Reis, Parati; São Paulo: Ubatuba, São Sebastião. TUNISIA. Tunis (Mediterrâneo).

Ocorrências novas são as de Ubatuba e Parati. Em São Paulo, a espécie era conhecida somente de São Sebastião, e agora foi visto ocorrer também em Ubatuba. Paracerceis sculpta deve ter invadido recentemente a costa sudeste brasileira, começando pelas praias próximas a porto (São Sebastião, p. ex.) e passando para as localidades vizinhas (Ubatuba). Em 1975, foram realizadas inúmeras coletas na região de Ubatuba, e P. sculpta nunca foi encontrada, embora atualmente seja uma espécie bastante comum na área.

\section{Material examinado}

Rio de Janeiro: Parati (Pr. do Guarda -Mor, em alga, entre-marés, 22.02.1982, R. Tsukamoto col., 1 ex.). São Paulo: Ubatuba (Enseada do Flamengo, Pr. do Lamberto, em Galaxaura sp., 1,5 m de prof. 10.10.1978, M. F1ynn col.,20exs.); São Sebastião (em placas de "fouling", 1-2 m de prof., 08.01.1981, S. Ditadi col., 8 exs.). Para lista completa do material examinado, ver Pires $1980 a$ e 1981a.

\section{Referências bibliogräficas}

BRUCE, N. L. 1980. The systematics of some Japanese marine isopods ( $\mathrm{fam}$. Sphaeromatidae) of the genera Dynoides Barnard, 1914 and Cymodocella Pfeffer, 1887, with descriptions of two new species. Crustaceana, 38(2): 199-211.

DOMMASNES, A. 1969. On the fauna of Corallina officinalis $\mathrm{L}$. in western Norway. Sarsia, 38:71-86.

GIAMBIAGI, D. 1922. Cuatro nuevos isōpodos de la Argentina. Physis, B Aires, 5:230-244.

HAGERMAN, L. 1966. The macro-and microfauna associated with Fucus serratus L., with some ecological remarks. Ophelia, 3:1-43.

HARRISON, K. \& HOLDICH, D. M. 1982. Revision of the genera Dynamenella, Ischyromene, Vynamenops is, and Cymodocella (Crustacea: Isopoda), including a new genus and five new species of Eubranchiate sphaeromatids from Queensland waters. J. Crust. Biol., 2(1):84-119.

HOLDICH, D. M. \& Harrison, K. 1981. The sphaeromatid isopod genus Sphaeromopsis Holdich \& Jones in African, Australian and South American waters. Crustaceana, 41 (3) : $286-300$.

\& Jones, D. A. 1973.

The systematics and ecology of a new genus of sand beach isopod

(Sphaeromatidae) from Kenya. J. Zool., Lond, , 171:385-395.

HOLMES, S. J. 1904. Remarks on the sexes of sphaeromids, with a description of a new species of Dynamene. Proc. Cal. Acad. Sci., 3(11): 296-304.

IVERSON, E. W. 1974. Range extensions for some California marine isopod crustaceans. Bull. Sth. Calif. Acad. Sci., 73(3): 164-169.

JANSEN, K. P. 1971. Ecological studies on interdital New Zealand Sphaeromatidae (Isopoda: Flabellifera). Mar,' Biol., 11:262-285.

LOYOLA E SILVA, J. de. 1959. Pseudosphaeroma jakobii n. sp. (Isopoda, Crustacea) encontrado na Baía de Guaratuba (Paranā-Brasil). Dusenia, 8(2):79-88. 
LOYOLA E SILVA, J. de 1960. Sphaeromatidae do litoral brasileiro. Bolm Univ. Paraná, Zoo1., 4:1-182.

1965. Espécie nova de Cymodocella Pfeffer, 1887 (Sphaeromatidae-Isopoda) do 1itoral brasileiro. Bolm Inst. Defesa Patrimônio nat., Secr. Agric., Zool. (7): $1-18$.

MENZIES, R. J. 1962. The marine isopod fauna of Bahia de San Quintin, Baja California, Mexico. Pac. Nat., 3(11): 237-348.

$$
\text { \& Glynn, P. W. } 1968 .
$$

The common marine isopod Crustacea of Puerto Rico. Uitg. Nat. Stud. Suriname, $27(51): 1-133$.

MUKAI, H. 1971. The phytal animals on the tha11i of Sargassum serratifolium in the Sargassum region, with reference to their seasonal fluctuations. Mar. Biol., 8(2):170-182.

PIRES, A. M. S. 1980a. Sergiella angra, a new genus and species of Sphaeromatidae (Isopoda) from Brazil. Crustaceana, 38(2):212-218.

\section{0b. New record of} Sphaeromatidae (Isopoda) from the Brazilian southern coast: Dynamenella dianae (Menzies, 1962). Crustaceana, $39(2): 133-140$.
PIRES, A. M. S. 1981a. Sergiella angra Pires, 1980, a junior synonym of Paracerceis sculpta (Holmes, 1904) (Isopoda, Sphaeromatidae). Crustaceana, 41 (2):219-220. 1981b. Ecologica1 study on littoral and infralittoral isopods from Ubatuba, Brazil. Bolm Inst. oceanogr., S Paulo, 30(1): $27-40$.

REZIG, M. 1978. Occurrence of Paracerceis sculpta (Crustacea, Isopoda, Flabellifera) in the lake of Tunis. Bu11. Off. Nat1. Peches (Tunisia), 2(1-2):175-191.

RICHARDSON, H. 1905. A monograph on the isopods of North America. Bull. U.S. natn. Mus., 54 :vii-liii $+1-727$.

1906. Descriptions of new isopod crustacean of the family Sphaeromidae. Proc. U.S. natn. Mus., $31: 1-22$.

1912. Description of a new species of isopod of the genus Cassidinidae from Mexico. Proc. U.S. natn. Mus., 42 (1886):107-108.

SCHULTZ, G. A, 1969. How to know the marine isopod crustaceans. Dubuque, Iowa, Wm. C. Brown Co. Pub., i-viii $+359 \mathrm{p}$.

(Manuscrito recebido em 08/Jun./1982; ace ito em 30/Nov./1982) 